\title{
Edge structures and properties of triangular antidots in single-layer $\mathrm{MoS}_{2}$
}

Li-Yong Gan', Yingchun Cheng', Udo Schwingenschlögl', Yingbang Yao, Yong Zhao, Xi-xiang Zhang, and Wei Huang

Citation: Appl. Phys. Lett. 109, 091603 (2016); doi: 10.1063/1.4962132

View online: http://dx.doi.org/10.1063/1.4962132

View Table of Contents: http://aip.scitation.org/toc/apl/109/9

Published by the American Institute of Physics 


\title{
Edge structures and properties of triangular antidots in single-layer $\mathrm{MoS}_{\mathbf{2}}$
}

\author{
Li-Yong Gan, 1,a) Yingchun Cheng, 2,a) Udo Schwingenschlögl, ${ }^{3, a)}$ Yingbang Yao, ${ }^{4,5}$ \\ Yong Zhao, ${ }^{1,6}$ Xi-xiang Zhang, ${ }^{3,4}$ and Wei Huang ${ }^{2}$ \\ ${ }^{1}$ Key Laboratory of Advanced Technology of Materials (Ministry of Education), Superconductivity and New \\ Energy R\&D Center, Southwest Jiaotong University, Chengdu, Sichuan 610031, China \\ ${ }^{2}$ Key Laboratory of Flexible Electronics (KLOFE) and Institute of Advanced Materials (IAM), \\ Jiangsu National Synergetic Innovation Center for Advanced Materials (SICAM), \\ Nanjing Tech University (NanjingTech), 30 South Puzhu Road, Nanjing 211816, China \\ ${ }^{3}$ Physical Science and Engineering Division (PSE), King Abdullah University of Science \\ and Technology (KAUST), Thuwal 23955-6900, Saudi Arabia \\ ${ }^{4}$ Advanced Nanofabrication and Imaging Core Lab, King Abdullah University of Science and Technology \\ (KAUST), Thuwal 23955-6900, Saudi Arabia \\ ${ }^{5}$ School of Materials and Energy, Guangdong University of Technology, Guangdong 510006, China \\ ${ }^{6}$ School of Physical Science and Technology, Southwest Jiaotong University, Chengdu, 610031 Sichuan, China
}

(Received 20 June 2016; accepted 20 August 2016; published online 30 August 2016)

\begin{abstract}
Density functional theory and experiments are employed to shed light on the edge structures of antidots in $\mathrm{O}$ etched single-layer $\mathrm{MoS}_{2}$. The equilibrium morphology is found to be the zigzag Mo edge with each Mo atom bonded to two $\mathrm{O}$ atoms, in a wide range of $\mathrm{O}$ chemical potentials. Scanning electron microscopy shows that the orientation of the created triangular antidots is opposite to the triangular shape of the single-layer $\mathrm{MoS}_{2}$ samples, in agreement with the theoretical predictions. Furthermore, edges induced by $\mathrm{O}$ etching turn out to be $p$-doped, suggesting an effective strategy to realize $p$-type $\mathrm{MoS}_{2}$ devices. Published by AIP Publishing. [http://dx.doi.org/10.1063/1.4962132]
\end{abstract}

Single-layer transition metal dichalcogenides are rapidly rising stars in the family of two-dimensional materials beyond graphene, being studied intensively due to exotic electronic, ${ }^{1-7}$ optical, ${ }^{8-10}$ mechanical, ${ }^{11-13}$ and chemical properties. ${ }^{14-18}$ In particular, single-layer $\mathrm{MoS}_{2}$ has a direct band gap of $\sim 2.0 \mathrm{eV}$ (Ref. 19) with high in-plane carrier mobility ${ }^{20,21}$ and on-off current ratio. ${ }^{2}$ It therefore performs excellently in electronic and optoelectonic devices ${ }^{22}$ as well as in indirect photocatalyzed hydrogen evolution reactions. ${ }^{23}$ Moreover, single-layer $\mathrm{MoS}_{2}$ shows huge spin splitting at the $\mathrm{K}$ and $\mathrm{K}^{\prime}$ valleys due to strong spin-orbit coupling and inversion symmetry breaking, ${ }^{24}$ thus forming an ideal template for exploring valleytronics and valley-dependent optoelectronics. ${ }^{25}$

Controlling the properties of single-layer $\mathrm{MoS}_{2}$ is important to meet the requirements of key applications. It has been demonstrated that an electric field, ${ }^{26}$ strain, ${ }^{27}$ defects, ${ }^{28}$ and the edge structure ${ }^{29}$ are effective means for introducing electronic and optical modulations. Specifically, the edge structure has been found to be crucial for optimizing the performance of epitaxial $\mathrm{MoS}_{2}$ nanosheets. The edge is also responsible for the catalytic activity in hydrogen evolution reactions ${ }^{30}$ which calls for maximizing its exposure by nanostructuring (nanoparticles ${ }^{31}$ or nanowires ${ }^{32}$ ). Recent experiments have introduced a strategy to fabricate internal edges, i.e., antidots, by heating $\mathrm{MoS}_{2}$ films in air. ${ }^{33,34}$ However, the exact edge structures have not yet been determined, while there exist pioneering theoretical studies exploring the electronic and magnetic properties of the antidots. ${ }^{35,36}$

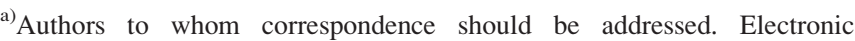
addresses: ganly@swjtu.edu.cn; iamyccheng@njtech.edu.cn; and
} udo.schwingenschlogl@kaust.edu.sa
Uncovering the edge morphology and properties of the antidots can provide new insights into ways to tailor the carrier type, Fermi level $\left(\mathrm{E}_{\mathrm{F}}\right)$, and catalytic activity. Antidots in single-layer $\mathrm{MoS}_{2}$ are likely to offer exciting opportunities to create new functionalities, as demonstrated in graphene. ${ }^{37}$ In this article, we therefore present a systematic study of the edge structures of single-layer $\mathrm{MoS}_{2}$ antidots using density functional theory combined with experiments. We show that the equilibrium morphology is the zigzag (ZZ) Mo edge saturated by $100 \% \mathrm{O}$, in a wide range of $\mathrm{O}$ potentials. This finding is verified by scanning electron microscopy (SEM). Examination of the electronic structure suggests that the $\mathrm{ZZ}$ Mo edge can provide shallow $p$-doping in single-layer $\mathrm{MoS}_{2}$.

First-principles calculations are performed using the Vienna Ab Initio Simulation Package with the spin polarized Perdew, Burke, and Ernzerhof generalized gradient approximation. For simulating antidots, we use a $12 \times 12 \times 1$ supercell with a large spacing of $15 \AA$ in the out-of-plane direction to create a two-dimensional model. We have checked that the spacing is sufficient to reduce interactions to a negligible level. A cutoff energy of $500 \mathrm{eV}$ and a $1 \times 1 \times 1 k$-mesh are used to optimize the structure until all residual forces remain below $0.02 \mathrm{eV} / \AA$ A , while a $\Gamma$-centered $2 \times 2 \times 1 k$-mesh is adopted for total energy and electronic structure calculations.

Experimentally, $\mathrm{MoS}_{2}$ is synthesized by the chemical vapor deposition method. We place high purity $\mathrm{MoO}_{3}(99.5 \%)$ and $\mathrm{S}$ powder $(99.5 \%)$ on a quartz boat and $\mathrm{Al}_{2} \mathrm{O}_{3}$ crucible in a hot-wall furnace, respectively. $\mathrm{A} \mathrm{SiO}_{2} / \mathrm{Si}$ substrate is placed on the quartz boat facing downwards. The reaction chamber is heated to $700^{\circ} \mathrm{C}$ with a rate of $15^{\circ} \mathrm{C} / \mathrm{min}$ in $\mathrm{Ar}$ flow $(10$ $\mathrm{sccm})$. Molybdenum oxide vapor reacts with sulfur vapor to grow $\mathrm{MoS}_{2}$ on the $\mathrm{SiO}_{2} / \mathrm{Si}$ substrate. The surface morphology is characterized by SEM (FEI Quanta 600). 
In order to calculate the energies of antidots with only $\mathrm{ZZ}$ or armchair (AC) Mo edges independently, triangularshaped antidots are used in our simulations. Figure 1(h) illustrates the antidot construction in a triangular Mo edge cluster, which is commonly created in S-rich conditions. ${ }^{29,38}$ The three edges of the grey triangle correspond to Mo edges of the $\mathrm{MoS}_{2}$ triangular samples typically observed in experiments. The orientation of the blue/red triangle is identical/ opposite to the gray triangle, illustrating the AC/ZZ Mo edge antidot. The edges can be unsaturated $(0 \%$ O coverage; Figs. $1(\mathrm{a})$ and $1(\mathrm{e}))$ or terminated with one $(50 \% \mathrm{O}$ coverage; Figs. 1(b), 1(c), and 1(f)) or two (100\% O coverage; Figs. $1(\mathrm{~d})$ and $1(\mathrm{~g})) \mathrm{O}$ atoms per edge Mo atom. For the $50 \% \mathrm{O}$ covered $\mathrm{ZZ}$ Mo edges, two situations are considered: $\mathrm{O}$ bonds are formed to one (50\%-I) or two (50\%-II) Mo atoms. For $100 \% \mathrm{O}$ coverage, the $\mathrm{O}$ atoms occupy $\mathrm{S}$ sites in the pristine $\mathrm{MoS}_{2}$ lattice.

In order to estimate the stability of the antidot edges at chemical potentials $\mu_{\mathrm{O}}$ of $\mathrm{O}, \mu_{\mathrm{S}}$ of $\mathrm{S}$, and $\mu_{\mathrm{Mo}}$ of Mo, the formation energy is defined as

$$
\Omega\left(N, \mu_{\mathrm{O}}\right)=E_{\mathrm{Mo}_{x} S_{y} \mathrm{O}_{z}}-E_{\mathrm{MoS}_{2}}+N_{\mathrm{Mo}} \mu_{\mathrm{Mo}}+N_{\mathrm{S}} \mu_{\mathrm{S}}-N_{\mathrm{O}} \mu_{\mathrm{O}},
$$

where the antidot size $N$ is given by the number of edge Mo atoms. For example, in Figs. 1(a)-1(d), we have $N=8$. $E_{\mathrm{Mo}_{x} S_{y} \mathrm{O}_{z}}$ and $E_{\mathrm{MoS}_{2}}$, respectively, are the total energies of the triangular antidot with the given edge type and stoichiometry and the ideal $12 \times 12 \times 1 \mathrm{MoS}_{2}$ single-layer. $N_{\mathrm{O}}, N_{\mathrm{S}}$, and $N_{\mathrm{Mo}}$ are the numbers of $\mathrm{O}, \mathrm{S}$, and Mo atoms added to or removed from the system, respectively. In thermodynamic equilibrium for $\mathrm{MoS}_{2}$ etching in O-rich conditions, $\mu_{\mathrm{S}}$ and $\mu_{\mathrm{Mo}}$ satisfy $\mu_{\mathrm{Mo}}+2 \mu_{\mathrm{O}}=E_{\mathrm{MoO}_{2}}^{\text {ref }}$ and $\mu_{\mathrm{S}}+2 \mu_{\mathrm{O}}=E_{\mathrm{SO}_{2}}^{\text {ref }}$, respectively, where $E_{\mathrm{MoO}_{2}}^{\text {ref }}$ and $E_{\mathrm{SO}_{2}}^{\text {ref }}$ are the total energies of a formula unit of $\mathrm{MoO}_{2}$ in its bulk phase and an isolated $\mathrm{SO}_{2}$ molecule. Then, Eq. (1) results in

$$
\begin{aligned}
\Omega\left(N, \mu_{\mathrm{O}}\right)= & E_{\mathrm{Mo}_{x} \mathrm{~S}_{\mathrm{y}} \mathrm{O}_{z}}-E_{\mathrm{MoS}_{2}}+N_{\mathrm{Mo}_{\mathrm{O}}} E_{\mathrm{MoO}_{2}}^{\mathrm{ref}}+N_{\mathrm{S}} E_{\mathrm{SO}_{2}}^{\mathrm{ref}} \\
& -\left(2 N_{\mathrm{Mo}}+2 N_{\mathrm{S}}+N_{\mathrm{O}}\right) \mu_{\mathrm{O}} .
\end{aligned}
$$

Similar to the approach of Ref. 39, the formation energy of a triangular antidot of size $N$ can be expressed as the edge

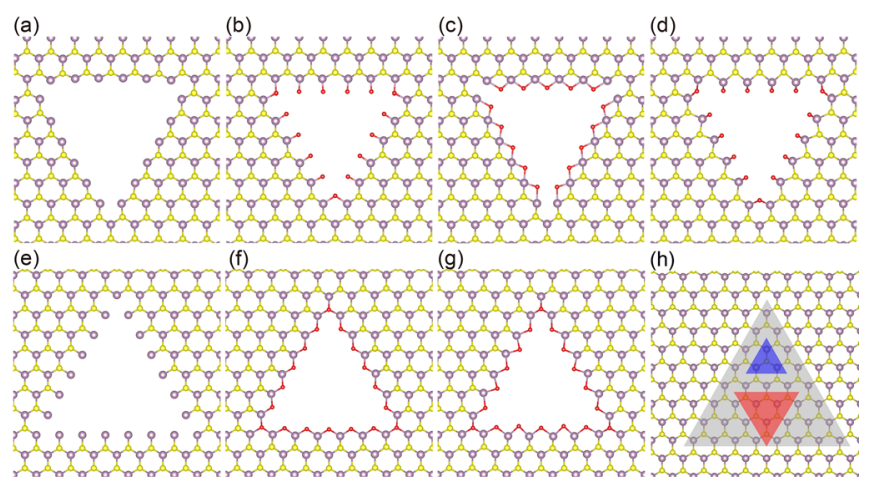

FIG. 1. Top views of triangular antidots: ZZ Mo edge with (a) $0 \%$, (b) $50 \%$ $\mathrm{I}$ (one $\mathrm{O}$ atom per Mo), (c) $50 \%$-II, and (d) $100 \% \mathrm{O}$ (two O atoms per Mo) coverage and AC Mo edge with (e) $0 \%$, (f) $50 \%$, and (g) $100 \%$ O coverage, after relaxation. (h) Construction of triangular ZZ Mo edge (red) and AC Mo edge (blue) antidots in a triangular Mo edge cluster (grey). The purple and yellow balls represent Mo and $\mathrm{S}$ atoms, respectively. energy, $\sigma\left(\mu_{\mathrm{O}}\right)$, times the number of edge atoms plus the corner energy, $\epsilon\left(\mu_{\mathrm{O}}\right)$, times the number of corner atoms

$$
\Omega\left(N, \mu_{\mathrm{O}}\right)=3(N-1) \sigma\left(\mu_{\mathrm{O}}\right)+3 \epsilon\left(\mu_{\mathrm{O}}\right) .
$$

When the size of the antidot is small, structural and/or electronic perturbations caused by a stoichiometry change in the system affect the total energy and thus $\sigma$ and $\varepsilon$. For large antidots, the total energy is less sensitive to perturbations and we can assume that $\sigma\left(\mu_{\mathrm{O}}\right)$ and $\epsilon\left(\mu_{\mathrm{O}}\right)$ do not depend on $N$. According to Eq. (2), $\Omega\left(N, \mu_{\mathrm{O}}=0\right)$ is plotted in Fig. 2 as a function of $N$ for the different edges. By linear fitting, edge $\left(\sigma_{\mathrm{O}}\right)$ and corner $\left(\epsilon_{\mathrm{O}}\right)$ energies are derived for each edge termination. The calculated antidot energies lie close to the fitting lines, validating Eq. (3), though small deviations are visible. These deviations possibly result from the stoichiometry (ratio of $x, y$, and $z$ in $\mathrm{Mo}_{x} \mathrm{~S}_{y} \mathrm{O}_{z}$ ) change with the antidot size, which affects the electronic states at the edge. The values $\sigma_{\mathrm{O}}$ and $\epsilon_{\mathrm{O}}$ are determined at $\mu_{\mathrm{O}}=0$. From Eq. (3), we obtain

$$
\Omega\left(N+1, \mu_{\mathrm{O}}\right)-\Omega\left(N, \mu_{\mathrm{O}}\right)=3 \sigma\left(\mu_{\mathrm{O}}\right)
$$

and

$$
\Omega(N+1,0)-\Omega(N, 0)=3 \sigma_{0} .
$$

Combination of Eqs. (4) and (5) with Eq. (2) leads to

$$
\sigma\left(\mu_{\mathrm{O}}\right)=\sigma_{0}-\frac{2\left[(N+1)_{\mathrm{Mo}}+(N+1)_{\mathrm{S}}-N_{\mathrm{Mo}}-N_{\mathrm{S}}\right]+\Delta N_{\mathrm{O}}}{3} \mu_{\mathrm{O}} .
$$

The term in square brackets describes the change in the number of Mo and $\mathrm{S}$ atoms between antidots of sizes $N$ and $N+1$, being $3 N-4$ and $3 N-8$, respectively, for ZZ and AC Mo edges. Moreover, $\Delta N_{\mathrm{O}}$ is the increment in the number of $\mathrm{O}$ atoms from $N$ to $N+1$, equaling 0,3 , and 6 for $0 \%, 50 \%$, and $100 \%$ O coverage, respectively. Consequently, we have

$$
\sigma\left(\mu_{\mathrm{O}}\right)=\sigma_{0}-\frac{6 N-8+\Delta N_{\mathrm{O}}}{3} \mu_{\mathrm{O}}
$$

and

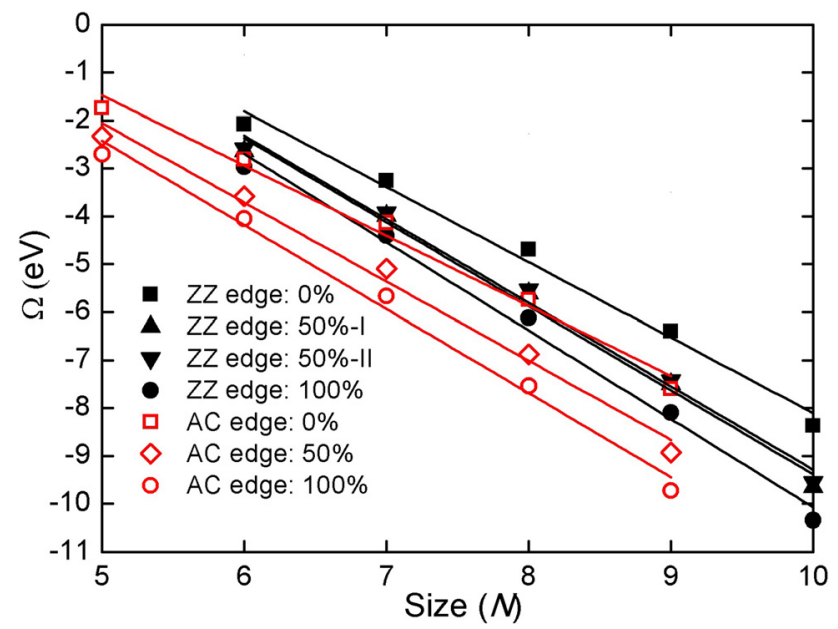

FIG. 2. Formation energy as a function of the antidot size at $\mu_{\mathrm{O}}=0$, according to Eq. (2). 


$$
\sigma\left(\mu_{\mathrm{O}}\right)=\sigma_{0}-\frac{6 N-16+\Delta N_{\mathrm{O}}}{3} \mu_{\mathrm{O}}
$$

for $\mathrm{ZZ}$ and AC Mo edges, respectively.

The formation energies of all antidots are negative, as can be seen in Fig. 2, indicating that it is feasible to create them by heating $\mathrm{MoS}_{2}$ in air. ${ }^{33,34}$ The $50 \%$-I configuration is energetically favorable over the $50 \%$-II configuration, which is rationalized by much stronger Mo-O bonding both at the corners and along the edges: The average Mo-O bond lengths of $1.91 \AA$ (corners) and $1.72 \AA$ (edges) in the 50\%-I configuration are much shorter than the corresponding values of $2.18 \AA$ and $1.97 \AA$ in the $50 \%$-II configuration. Bader charge analysis, ${ }^{40}$ which is an effective tool to calculate the charge on individual atoms in molecules as well as crystals, indicates that both the corner and edge Mo atoms lose more charge in the 50\%-I configuration, which lowers the total energy. Thus, only this configuration is considered for $50 \%$ $\mathrm{O}$ coverage in the following. We note that the theoretical results in this work are based on freestanding single-layer $\mathrm{MoS}_{2}$ and do not consider the substrate effects present in the experiment. Nonetheless, the interaction between singlelayer $\mathrm{MoS}_{2}$ and the substrate, such as $\mathrm{SiO}_{2} / \mathrm{Si}^{3},{ }^{3,34}$ is mainly of van der Waals type ${ }^{41}$ and thus has little effect on the edge energies of the antidots.

Figure 3 displays the edge energy as a function of $\Delta \mu_{\mathrm{O}}=\mu_{\mathrm{O}}-\frac{1}{2} E_{\mathrm{O}_{2}}^{\text {total }}$, which is zero in O-rich conditions. At high temperature, the Mo and $\mathrm{S}$ atoms are converted into $\mathrm{MoO}_{3}$ and $\mathrm{SO}_{2}$ gas molecules, ${ }^{33,34}$ the latter resulting in higher $\Delta \mu_{\mathrm{O}}$ in O-poor conditions. Thus, $\Delta \mu_{\mathrm{O}}=-\frac{1}{2} H_{\mathrm{SO}_{2}}^{\mathrm{f}}$ in this case, where $H_{\mathrm{SO}_{2}}^{\mathrm{f}}$ is the formation energy of a $\mathrm{SO}_{2}$ molecule. The straight lines in Fig. 3 are given by Eqs. (5) and (6). In a wide range of $\mathrm{O}$ conditions $\left(\Delta \mu_{\mathrm{O}}\right.$ from -1.5 to $0 \mathrm{eV}$ ), the $\mathrm{ZZ}$ Mo edge with $100 \%$ O coverage has the lowest energy, followed by the 50\%-I ZZ Mo edge, and the AC Mo edge with $100 \%$ O coverage. In O-rich conditions, the 50\%-I ZZ Mo edge is energetically more favorable over the AC Mo edge with $100 \% \mathrm{O}$ coverage. Thus, in $\mathrm{O}$ flow ${ }^{34}$ the $\mathrm{ZZ} \mathrm{Mo}$ edge is terminated by an $\mathrm{O}$ dimer, shown in Fig. 1(d), and the AC edge has $100 \% \mathrm{O}$ coverage with the structure shown in Fig. 1(g) (much larger energy than the ZZ Mo edge). These results agree well with previous experimental

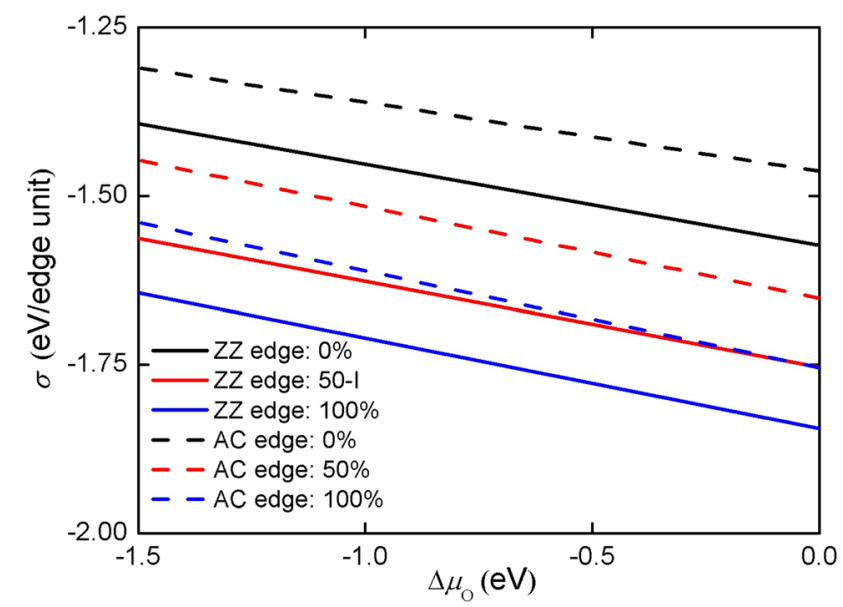

FIG. 3. Edge energy as a function of the $\mathrm{O}$ chemical potential. The antidot size is set to $N=10$. observations that the edges are of $\mathrm{ZZ}$ Mo type. ${ }^{33,34}$ Furthermore, based on our findings, the experimental edges are likely to have $100 \%$ O coverage and to exhibit O dimer termination in the O-rich environment.

Once the edge energies are obtained, the equilibrium edge morphology can be determined by applying the Wulff construction rule, ${ }^{42}$ which is given by $\frac{\sigma_{\mathrm{ZZ}}}{d_{\mathrm{ZZ}}}=\frac{\sigma_{\mathrm{AC}}}{d_{\mathrm{AC}}}$, where $\sigma_{\mathrm{ZZ}}$ $\left(\sigma_{\mathrm{AC}}\right)$ is the minimum surface energy according to Fig. 3 for the $\mathrm{ZZ}$ (AC) Mo edge and $d_{\mathrm{ZZ}}\left(d_{\mathrm{AC}}\right)$ is the distance of the $\mathrm{ZZ}$ (AC) Mo edge to the center of the antidot. In the whole $\mathrm{O}$ chemical potential range $\left(\Delta \mu_{\mathrm{O}}\right.$ from -1.5 to $\left.0 \mathrm{eV}\right)$, the morphology is dominated by the ZZ Mo edge with the edge Mo atoms bonded to $\mathrm{O}$ dimers, shown in Fig. 4(a). The average Mo-O bond length and $\mathrm{O}-\mathrm{O}$ distance at the corners are 1.96 and $2.45 \AA$, respectively, while the corresponding values along the edges are 1.74 and $2.66 \AA$. This is much less than the Mo-S bond length $(2.41 \AA)$ and $\mathrm{S}$-S distance $\left(3.13 \AA\right.$ ) in pristine single-layer $\mathrm{MoS}_{2}$. Specifically, the Mo atoms bonded to edge $\mathrm{O}$ atoms protrude (in-plane) by $0.51 \AA$, as shown by the black lines in Fig. 4(a).

A simulated scanning tunneling microscopy image of the ZZ Mo edge terminated by $\mathrm{O}$ dimers $(N=8)$ is shown in Fig. 4(b). We observe two bright triangular features around the antidot, whereas the other regions appear dark. The bright areas are likely to serve as active sites due to high charge density, which facilitates charge transfer to the reactant molecules. ${ }^{43}$ The inner bright triangle corresponds to the $\mathrm{O}$ atoms, with more intensity at the corners $\left(\mathrm{O} p_{\mathrm{z}}\right.$ contributions) than along the edges $\left(\mathrm{O} p_{\mathrm{x}} / p_{\mathrm{y}}\right.$ contributions $)$, and the outer triangle on the $\mathrm{S}$ atoms, displaying $p_{\mathrm{x}} / p_{\mathrm{y}}$ features. The intensity decreases gradually from the edges to the corners due to a structural effect: The protrusion of the edge Mo atoms weakens their bonding to the edge $\mathrm{S}$ atoms.

To validate our predictions, SEM is applied to a singlelayer $\mathrm{MoS}_{2}$ sample after exposure to air at $100^{\circ} \mathrm{C}$ for $10 \mathrm{~min}$. Figure 5(a) shows an optical image of the as-prepared sample. We observe a triangular shape with ZZ S edges. ${ }^{29,38}$ According to the Raman spectrum of the as-prepared sample in Fig. 5(b), the Raman shifts of the $\mathrm{E}_{2 \mathrm{~g}}$ and $\mathrm{A}_{1 \mathrm{~g}}$ modes are 386.3 and $407.2 \mathrm{~cm}^{-1}$. The difference of $20.9 \mathrm{~cm}^{-1}$ indicates that sample is single-layered. ${ }^{44}$ The samples show cracks after heating, see Fig. 5(b), possibly due to fast cooling. Additionally, distinct triangular antidots can be observed in the zoomed SEM images in Figs. 5(c) and 5(d), demonstrating that heating in air is an effective way to create such

(a)
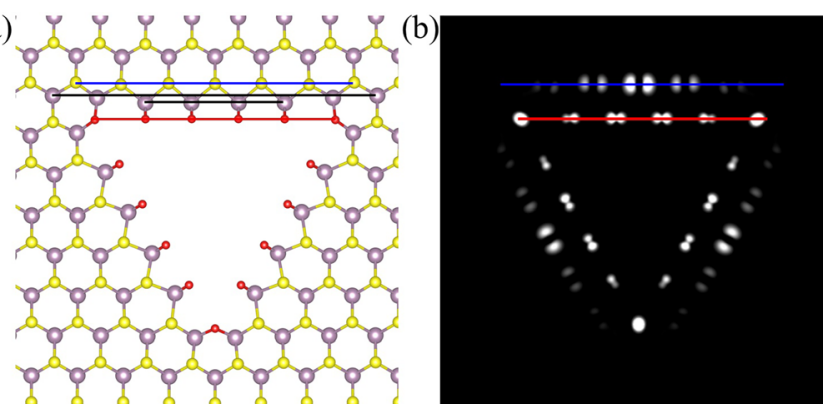

FIG. 4. ZZ Mo edge with 100\% O coverage: (a) structure and (b) simulated scanning tunneling microscopy image at a voltage of $-0.3 \mathrm{~V}$. Black lines highlight the protrusion of the edge Mo atoms. Blue and red lines refer the characteristic features in (b) to the atomic structure in (a). 


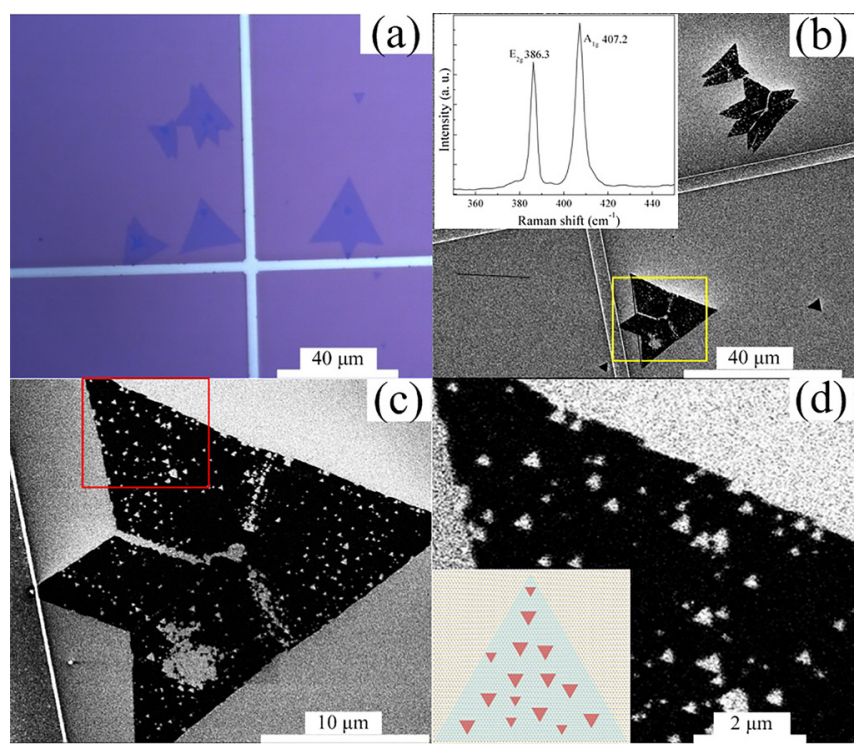

FIG. 5. (a) Optical image of the as-prepared single-layer $\mathrm{MoS}_{2}$. (b) SEM image of the same region after exposure to air at $100^{\circ} \mathrm{C}$ for $10 \mathrm{~min}$. The inset shows the Raman spectrum of the as-prepared sample. (c) Zoomed view of the yellow rectangle in (b). (d) Zoomed view of the red rectangle in (c). The inset depicts the orientation of the triangular antidots (red) with respect to the triangular single-layer $\mathrm{MoS}_{2}$ sample (grey).

antidots. The inset in Fig. 5(d) reveals that the antidots are oriented oppositely to the triangular shape of the single-layer $\mathrm{MoS}_{2}$ samples, which suggests that we have ZZ Mo edges according to our theoretical prediction.

To explore potential applications, total densities of states of a $\mathrm{MoS}_{2}$ single-layer with $\mathrm{ZZ}$ and AC Mo edge antidots covered by $100 \% \mathrm{O}$ are shown in Fig. 6 as a function of the antidot size. The main features of the band edges are largely maintained, while defect states appear in the band gap. For the $\mathrm{ZZ}$ and $\mathrm{AC}$ Mo edge antidots, respectively, $\mathrm{E}_{\mathrm{F}}$ is shifted from the middle of the band gap (ideal single-layer $\mathrm{MoS}_{2}$ ) towards the valence and conduction band, which
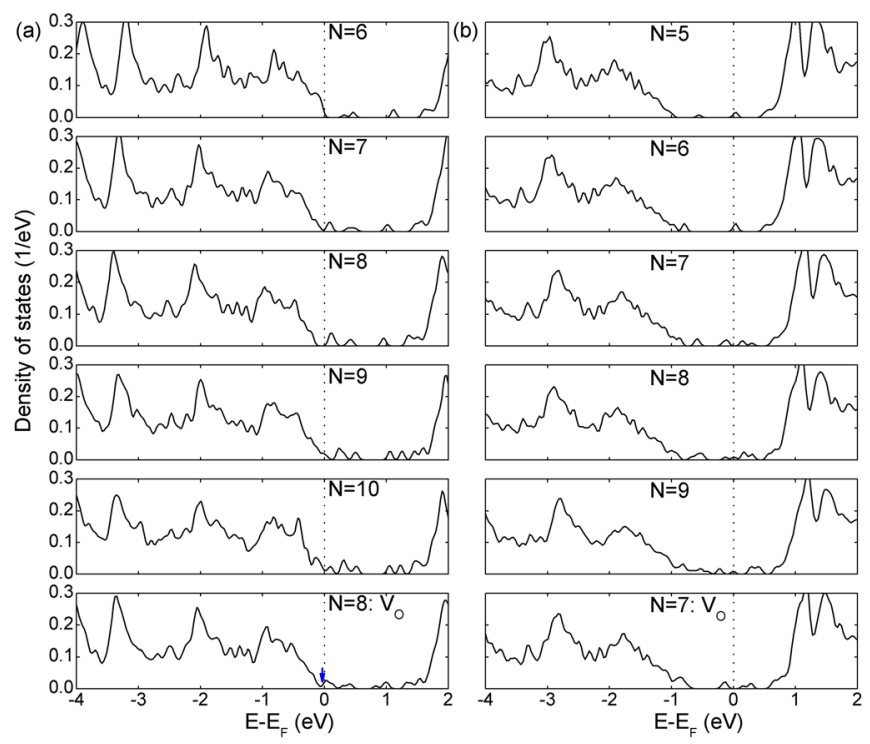

FIG. 6. Size-dependence of the density of states of (a) ZZ and (b) AC Mo edge antidots with $100 \% \mathrm{O}$ coverage. The energy zero is $E_{F}$. The effect of an $\mathrm{O}$ vacancy located on the edge is addressed in the bottom panels. A blue arrow indicates the induced defect states. reflects $p$ - and $n$-doping. Since in the former case, $\mathrm{E}_{\mathrm{F}}$ is located closer to the band edge, the $p$-doping is shallower than the $n$-doping. Increasing the size of the antidots enhances the doping effects and gradually gives rise to metallic features, as shown by the finite density of states at $\mathrm{E}_{\mathrm{F}}$ for $N=10$. In addition, we find that $\mathrm{O}$ vacancies along the edge are energetically strongly favorable over those at the corners, for example, -0.68 versus $1.47 \mathrm{eV}$ for the $N=8 \mathrm{ZZ} \mathrm{Mo}$ edge antidot. The negative value suggests that $O$ vacancies are likely to exist along the edge. $\mathrm{O}$ vacancies induce hole states at the valence band edge as shown by the blue arrow in Fig. 6, and therefore enhance the $p$-doping. It has been reported that $n$-type single-layer $\mathrm{MoS}_{2}$ transistors can be fabricated with relative ease, while realization of $p$-type devices remains challenging. ${ }^{44}$ Our results establish not only the edge structures of $\mathrm{O}$ etched antidots but also suggest that $\mathrm{O}$ etching is able to $p$-dope single-layer $\mathrm{MoS}_{2}$. In O-rich conditions, the antidot will grow with time, especially at higher temperature.

We have investigated the edge structures of antidots in single-layer $\mathrm{MoS}_{2}$ using a combination of calculations based on density functional theory and SEM experiments. The theoretical edge energies reveal that the equilibrium morphology is likely the ZZ Mo edge with $100 \%$ O coverage. This prediction is verified by SEM, which also shows that the triangular antidots are oriented oppositely to the triangular single-layer $\mathrm{MoS}_{2}$ samples. Further examination of the electronic structure suggests that $\mathrm{O}$ etching is able to $p$-dope single-layer $\mathrm{MoS}_{2}$.

This work was supported by the National Natural Science Foundation of China (Nos. 11504303, 11504169, 61575094, and 61136003), the National Basic Research Program of China (2015CB932200), the Fundamental Research Funds for the Central Universities (SWJTU2682016ZDPY10), and the Synergetic Innovation Center for Organic Electronics and Information Displays. The research reported in this publication was supported by funding from King Abdullah University of Science and Technology (KAUST).

${ }^{1}$ J. V. Lauritsen, J. Kibsgaard, S. Helveg, H. Topsoe, B. S. Clausen, E. Laegsgaard, and F. Besenbacher, "Size-dependent structure of $\mathrm{MoS}_{2}$ nanocrystals," Nat. Nanotechnol. 2(1), 53-58 (2007).

${ }^{2}$ B. Radisavljevic, A. Radenovic, J. Brivio, V. Giacometti, and A. Kis, "Single-layer $\mathrm{MoS}_{2}$ transistors," Nat. Nanotechnol. 6(3), 147-150 (2011). ${ }^{3}$ Y. Y. Hui, X. Liu, W. Jie, N. Y. Chan, J. Hao, Y.-T. Hsu, L.-J. Li, W. Guo, and S. P. Lau, "Exceptional tunability of band energy in a compressively strained trilayer $\mathrm{MoS}_{2}$ sheet," ACS Nano 7(8), 7126-7131 (2013).

${ }^{4}$ M. Chhowalla, H. S. Shin, G. Eda, L.-J. Li, K. P. Loh, and H. Zhang, "The chemistry of two-dimensional layered transition metal dichalcogenide nanosheets," Nat. Chem. 5(4), 263-275 (2013).

${ }^{5}$ M.-Y. Li, Y. Shi, C.-C. Cheng, L.-S. Lu, Y.-C. Lin, H.-L. Tang, M.-L. Tsai, C.-W. Chu, K.-H. Wei, J.-H. He, W.-H. Chang, K. Suenaga, and L.J. Li, "Epitaxial growth of a monolayer $\mathrm{WSe}_{2}-\mathrm{MoS}_{2}$ lateral p-n junction with an atomically sharp interface," Science 349(6247), 524-528 (2015).

${ }^{6} \mathrm{X}$. Xi, L. Zhao, Z. Wang, H. Berger, L. Forró, J. Shan, and K. F. Mak, "Strongly enhanced charge-density-wave order in monolayer $\mathrm{NbSe}_{2}$," Nat. Nanotechnol. 10(9), 765-769 (2015).

${ }^{7}$ X. Duan, C. Wang, Z. Fan, G. Hao, L. Kou, U. Halim, H. Li, X. Wu, Y. Wang, J. Jiang, A. Pan, Y. Huang, R. Yu, and X. Duan, "Synthesis of $\mathrm{WS}_{2 \mathrm{x}} \mathrm{Se}_{2-2 \mathrm{x}}$ alloy nanosheets with composition-tunable electronic properties," Nano Lett. 16(1), 264-269 (2016).

${ }^{8}$ Z. Yin, H. Li, H. Li, L. Jiang, Y. Shi, Y. Sun, G. Lu, Q. Zhang, X. Chen, and H. Zhang, "Single-layer $\mathrm{MoS}_{2}$ phototransistors," ACS Nano 6(1), 74-80 (2012). 
${ }^{9}$ K. F. Mak, K. He, C. Lee, G. H. Lee, J. Hone, T. F. Heinz, and J. Shan, "Tightly bound trions in monolayer $\mathrm{MoS}_{2}$," Nat. Mater. 12(3), 207-211 (2013).

${ }^{10}$ A. F. Rigosi, H. M. Hill, Y. Li, A. Chernikov, and T. F. Heinz, "Probing interlayer interactions in transition metal dichalcogenide heterostructures by optical spectroscopy: $\mathrm{MoS}_{2} / \mathrm{WS}_{2}$ and $\mathrm{MoSe}_{2} / \mathrm{WSe}_{2}$," Nano Lett. 15(8), 5033-5038 (2015).

${ }^{11} \mathrm{~S}$. Bertolazzi, J. Brivio, and A. Kis, "Stretching and breaking of ultrathin $\mathrm{MoS}_{2}$," ACS Nano 5(12), 9703-9709 (2011).

${ }^{12}$ J. Pu, Y. Yomogida, K.-K. Liu, L.-J. Li, Y. Iwasa, and T. Takenobu, "Highly flexible $\mathrm{MoS}_{2}$ thin-film transistors with ion gel dielectrics," Nano Lett. 12(8), 4013-4017 (2012).

${ }^{13}$ A. Castellanos-Gomez, M. Poot, G. A. Steele, H. S. J. van der Zant, N. Agrait, and G. Rubio-Bollinger, "Elastic properties of freely suspended $\mathrm{MoS}_{2}$ nanosheets," Adv. Mater. 24(6), 772-775 (2012).

${ }^{14}$ M. A. Lukowski, A. S. Daniel, F. Meng, A. Forticaux, L. Li, and S. Jin, "Enhanced hydrogen evolution catalysis from chemically exfoliated metallic $\mathrm{MoS}_{2}$ nanosheets,” J. Am. Chem. Soc. 135(28), 10274-10277 (2013).

${ }^{15}$ D. Voiry, H. Yamaguchi, J. Li, R. Silva, D. C. B. Alves, T. Fujita, M. Chen, T. Asefa, V. B. Shenoy, G. Eda, and M. Chhowalla, "Enhanced catalytic activity in strained chemically exfoliated $\mathrm{WS}_{2}$ nanosheets for hydrogen evolution," Nat. Mater. 12(9), 850-855 (2013).

${ }^{16} \mathrm{C}$. Tsai, F. Abild-Pedersen, and J. K. Nørskov, "Tuning the $\mathrm{MoS}_{2}$ edge-site activity for hydrogen evolution via support interactions," Nano Lett. 14(3), 1381-1387 (2014).

${ }^{17}$ H. Li, C. Tsai, A. L. Koh, L. Cai, A. W. Contryman, A. H. Fragapane, J. Zhao, H. S. Han, H. C. Manoharan, F. Abild-Pedersen, J. K. Norskov, and $\mathrm{X}$. Zheng, "Activating and optimizing $\mathrm{MoS}_{2}$ basal planes for hydrogen evolution through the formation of strained sulphur vacancies," Nat. Mater. 15(1), 48-53 (2016).

${ }^{18}$ M. Asadi, B. Kumar, A. Behranginia, B. A. Rosen, A. Baskin, N. Repnin, D. Pisasale, P. Phillips, W. Zhu, R. Haasch, R. F. Klie, P. Král, J. Abiade, and A. Salehi-Khojin, "Robust carbon dioxide reduction on molybdenum disulphide edges," Nat. Commun. 5, 4470 (2014).

${ }^{19}$ Y. Chen, J. Xi, D. O. Dumcenco, Z. Liu, K. Suenaga, D. Wang, Z. Shuai, Y.-S. Huang, and L. Xie, "Tunable band gap photoluminescence from atomically thin transition-metal dichalcogenide alloys," ACS Nano 7(5), 4610-4616 (2013).

${ }^{20}$ H. Liu, A. T. Neal, and P. D. Ye, "Channel length scaling of $\mathrm{MoS}_{2}$ MOSFETs,” ACS Nano 6(10), 8563-8569 (2012).

${ }^{21}$ D. Jariwala, V. K. Sangwan, D. J. Late, J. E. Johns, V. P. Dravid, T. J. Marks, L. J. Lauhon, and M. C. Hersam, "Band-like transport in high mobility unencapsulated single-layer $\mathrm{MoS}_{2}$ transistors," Appl. Phys. Lett. 102(17), 173107 (2013).

${ }^{22}$ Q. H. Wang, K. Kalantar-Zadeh, A. Kis, J. N. Coleman, and M. S. Strano, "Electronics and optoelectronics of two-dimensional transition metal dichalcogenides," Nat. Nanotechnol. 7(11), 699-712 (2012).

${ }^{23}$ D. Deng, K. S. Novoselov, Q. Fu, N. Zheng, Z. Tian, and X. Bao, "Catalysis with two-dimensional materials and their heterostructures," Nat. Nanotechnol. 11(3), 218-230 (2016).

${ }^{24}$ Z. Y. Zhu, Y. C. Cheng, and U. Schwingenschlögl, "Giant spin-orbitinduced spin splitting in two-dimensional transition-metal dichalcogenide semiconductors," Phys. Rev. B 84(15), 153402 (2011).

${ }^{25}$ H. Zeng, J. Dai, W. Yao, D. Xiao, and X. Cui, "Valley polarization in $\mathrm{MoS}_{2}$ monolayers by optical pumping," Nat. Nanotechnol. 7(8), 490-493 (2012).

${ }^{26}$ S. Wu, J. S. Ross, G.-B. Liu, G. Aivazian, A. Jones, Z. Fei, W. Zhu, D. Xiao, W. Yao, D. Cobden, and X. Xu, "Electrical tuning of valley magnetic moment through symmetry control in bilayer $\mathrm{MoS}_{2}$," Nat. Phys. 9(3), 149-153 (2013).
${ }^{27}$ J. Feng, X. Qian, C.-W. Huang, and J. Li, "Strain-engineered artificial atom as a broad-sectrum solar energy funnel," Nat. Photonics 6(12), 866-872 (2012).

${ }^{28}$ W. Zhou, X. Zou, S. Najmaei, Z. Liu, Y. Shi, J. Kong, J. Lou, P. M. Ajayan, B. I. Yakobson, and J.-C. Idrobo, "Intrinsic structural defects in monolayer molybdenum disulfide," Nano Lett. 13(6), 2615-2622 (2013).

${ }^{29}$ D. Cao, T. Shen, P. Liang, X. Chen, and H. Shu, "Role of chemical potential in flake shape and edge properties of monolayer $\mathrm{MoS}_{2}$," J. Phys. Chem. C 119(8), 4294-4301 (2015).

${ }^{30}$ T. F. Jaramillo, K. P. Jørgensen, J. Bonde, J. H. Nielsen, S. Horch, and I. Chorkendorff, "Identification of active edge sites for electrochemical $\mathrm{H}_{2}$ evolution from $\mathrm{MoS}_{2}$ nanocatalysts," Science 317(5834), 100-102 (2007).

${ }^{31}$ Y. Li, H. Wang, L. Xie, Y. Liang, G. Hong, and H. Dai, "MoS $\mathrm{M}_{2}$ nanoparticles grown on graphene: An advanced catalyst for the hydrogen evolution reaction," J. Am. Chem. Soc. 133(19), 7296-7299 (2011).

${ }^{32}$ Z. Chen, D. Cummins, B. N. Reinecke, E. Clark, M. K. Sunkara, and T. F. Jaramillo, "Core-shell $\mathrm{MoO}_{3}-\mathrm{MoS}_{2}$ nanowires for hydrogen evolution: A functional design for electrocatalytic materials," Nano Lett. 11(10), 4168-4175 (2011).

${ }^{33}$ H. Zhou, F. Yu, Y. Liu, X. Zou, C. Cong, C. Qiu, T. Yu, Z. Yan, X. Shen, L. Sun, B. I. Yakobson, and J. M. Tour, "Thickness-dependent patterning of $\mathrm{MoS}_{2}$ sheets with well-oriented triangular pits by heating in air," Nano Res. 6(10), 703-711 (2013).

${ }^{34}$ R. Ionescu, A. George, I. Ruiz, Z. Favors, Z. Mutlu, C. Liu, K. Ahmed, R. Wu, J. S. Jeong, L. Zavala, K. A. Mkhoyan, M. Ozkan, and C. S. Ozkan, "Oxygen etching of thick $\mathrm{MoS}_{2}$ films," Chem. Commun. 50(76), 11226-11229 (2014).

${ }^{35}$ Y. Zhou, P. Yang, H. Zu, F. Gao, and X. Zu, "Electronic structures and magnetic properties of $\mathrm{MoS}_{2}$ nanostructures: Atomic defects, nanoholes, nanodots and antidots," Phys. Chem. Chem. Phys. 15(25), 10385-10394 (2013).

${ }^{36}$ L. Shao, G. Chen, H. Ye, Y. Wu, H. Niu, and Y. Zhu, "Theoretical study on electronic properties of $\mathrm{MoS}_{2}$ antidot lattices," J. Appl. Phys. 116(11), 113704 (2014).

${ }^{37}$ T. G. Pedersen, C. Flindt, J. Pedersen, N. A. Mortensen, A.-P. Jauho, and K. Pedersen, "Graphene antidot lattices: Designed defects and spin qubits," Phys. Rev. Lett. 100(13), 136804 (2008).

${ }^{38}$ H. Schweiger, P. Raybaud, G. Kresse, and H. Toulhoat, "Shape and edge sites modifications of $\mathrm{MoS}_{2}$ catalytic nanoparticles induced by working conditions: A theoretical study," J. Catal. 207(1), 76-87 (2002).

${ }^{39}$ S. Helveg, J. V. Lauritsen, E. Laegsgaard, I. Stensgaard, J. K. Norskov, B. S. Clausen, H. Topsoe, and F. Besenbacher, "Atomic-scale structure of single-layer $\mathrm{MoS}_{2}$ nanoclusters," Phys. Rev. Lett. 84(5), 951-954 (2000).

${ }^{40} \mathrm{R}$. Bader, Atoms in Molecules: A Quantum Theory (Oxford University, New York, 1990).

${ }^{41}$ Y. Cheng, K. Yao, Y. Yang, L. Li, Y. Yao, Q. Wang, X. Zhang, Y. Han, and U. Schwingenschloegl, "Van der Waals epitaxial growth of $\mathrm{MoS}_{2}$ on $\mathrm{SiO}_{2} / \mathrm{Si}$ by chemical vapor deposition," RSC Adv. 3(38), 17287-17293 (2013).

${ }^{42} \mathrm{G}$. Z. Wulff, "On the question of the rate of growth and dissolution of crystal surfaces," Kristallogr. Mineral., Tr. Fedorovskoi Yubileinoi Sess. 34, 449-530 (1901).

${ }^{43}$ F. Li, H. Shu, C. Hu, Z. Shi, X. Liu, P. Lang, and X. Chen, "Atomic mechanism of electrocatalytically active $\mathrm{Co}-\mathrm{N}$ complexes in graphene basal plane for oxygen reduction reaction," ACS Appl. Mater. Interfaces 7(49), 27405-27413 (2015).

${ }^{44}$ C. Lee, H. Yan, L. E. Brus, T. F. Heinz, J. Hone, and S. Ryu, “Anomalous lattice vibrations of single- and few-layer $\mathrm{MoS}_{2}$," ACS Nano 4(5), 2695-2700 (2010). 